\title{
Socio-Technical Drivers for Community Renewable Energy Systems - Analysis of Case Studies from India
}

\author{
Sutapa Pati ${ }^{1}$ \\ ${ }^{1}$ Xavier School of Sustainability, Xavier University, Bhubaneswar, India \\ Correspondence: Dr. Sutapa Pati, Associate Professor, Xavier School of Sustainability, Xavier University, \\ Bhubaneswar, India. E-mail: sutapa.pati@gmail.com
}

Received: March 30, 2017

Accepted: April 17, $2017 \quad$ Online Published: May 31, 2017

doi:10.5539/jsd.v10n3p143

URL: https://doi.org/10.5539/jsd.v10n3p143

\begin{abstract}
Renewable energy sector of India has the potential to be a credible alternative for households and communities. The sector accounts for approximately 13 per cent of the national energy capacity, including technologies such as solar grid/ off - grid generation, wind power, small hydro power, biomass/ biogas, and waste to energy. Statistics related to implementation illustrate the scope of tapping the renewable energy sector comprehensively, esp. for decentralized, household and community level applications. This paper analyses on - ground case studies and projects from various renewable energy categories, specifically the socio - technical and community aspects that play a significant role in successful Renewable Energy Technologies (henceforth RET) implementation. The distilled positive and negative drivers include technical, economic considerations, policy and regulatory, environmental aspects, market/ business models, and social/ community linked aspects. Case studies describe the need for a system based and stakeholder engagement approach where all possible stakeholders and their issues are envisaged and taken into account. Use of an evaluating framework is suggested to ensure successful community models of RETs and mass adoption of renewable technologies.
\end{abstract}

Keywords: community renewable energy system, renewable energy technology, off-grid, socio-technical barriers \& drivers

\section{Introduction}

\subsection{India’s Energy Security Outlook}

The United Nations launched Sustainable Energy for All Initiative with the overall objective of attaining universal energy access by the year 2030. In this context, India's energy scenario and future projections assume significance. India's current energy mix is highly skewed towards fossil fuels, with renewable sources accounting for $13 \%$ installed power generation and $4 \%$ of the energy mix (see Table 1). According to Central Electricity Authority (CEA, 2016), the overall installed capacities of various modes of power generation are given as below.

Table 1. Total installed power generation capacity in India as of $29^{\text {th }}$ February 2016

\begin{tabular}{lll}
\hline Fuel & MW & (\%) Age \\
\hline Total Thermal & 201360.04 & 69.8 \\
Coal & 175857.88 & 60.9 \\
Gas & 24508.63 & 8.5 \\
Diesel & 993.53 & 0.3 \\
Hydro & 42703.42 & 14.8 \\
Nuclear & 5780.00 & 2.0 \\
Renewable energy sources & 38821.51 & 13.4 \\
\hline Total & $\mathbf{2 8 8 6 6 4 . 9 7}$ & $\mathbf{1 0 0 . 0}$ \\
\hline
\end{tabular}

Source: Central Electricity Authority (CEA), 2016 
As seen from Table 1, close to 70 per cent of India's electricity generation capacities are dependent on coal and natural gas. Sargsyan, Bhatia, Banerjee, and Raghunathan (2011) estimated that India's coal reserves shall last only another 45 years if current usage rates were maintained. Projections, however, suggest higher per capita energy consumption and appliance usage in coming decades (World Bank, 2008; Bhattacharyya, 2015). Globally, India is the third largest energy consumer at present - by 2035, the energy use pattern is expected to increase by 3-4 times, while its' population is projected to cross 1.5 billion (A., Kumar, K., Kumar, Kaushik, Sharma, \& Mishra, 2010).

Energy access and security issues are likely to extend in short - term future. As per International Energy Agency (IEA, 2012) estimates, approximately 150 million persons across India will not have access to electricity even by 2030 (cited in Bhattacharyya, Palit, \& Kishore, 2014). As per existing scenario, energy access in rural areas has been estimated for 95.6 percent of Indian villages (CEA, 2014; Palit, Garimella, Shardul, \& Chaudbury, 2015) though household electricity coverage has only reached an upper level of $67 \%$ (Census of India, 2011). Close to 700 million persons use traditional biomass sources for cooking (e.g., dung, crop waste and firewood) (Smith \& Sagar, 2014), and further, 77.5 million households (approximately 400 million persons) rely on kerosene for domestic lighting (Census of India, 2011).

Energy poverty and access issues in India are predominant in rural areas on account of lack of grid extension and affordability. Accordingly, the Government of India set up the Rural Electricity Supply Technology (REST) Mission (2001) with a target of 'power for all' by 2012. This was followed by the consequent launch of Rajiv Gandhi Grameen Vidyutikaran Yojana (RGGVY). Outreach under RGGVY was estimated to be 23.67 million households, of which 21.27 million were said to be Below Poverty Line (BPL) households (Balachandra 2013). In recent years, rural electrification has received a push through multiple schemes and programmes (see Samanta 2015 for various schemes and programmes related to village electrification) including Deendayal Upadhyaya Grameen Vidyutikaran Yojana (DDUGVKY, earlier RGGVY) \& Remote Village Electrification Programme (RVEP) (note 1). Government of India has set the target for electrification of all remaining villages by 2018 (GoI, 2017). This, however, needs to be understood in light of the currently accepted definition of electrification in India, which is considerably limited by international standards (note 2). Policy wise, a minimum benchmark of consumption of one unit/ household / day as a merit good was established in the 2005 National Electrification Policy (MoP, 2017). Hence, achieving complete electrification may not assuredly provide energy security to all households. Further, access to electricity itself may not be adequate in terms of fulfilling quality aspects, as supply remains poor in rural areas and during night hours when needed (Palit \& Chaurey, 2011).

This paper analyzes community renewable energy systems as a viable alternative for ensuring energy access and security. Renewable energy systems across the scale have been implemented in India (section 1.2). The scope for community energy systems remains promising in the context of commercially viable technologies, a developing business ecosystem, locally relevant renewable resources and a productive rather than minimalistic approach to energy generation. Distributed generation, or off-grid renewable technologies have not yet been scaled up to the optimized potential due to multiple factors such as post - implementation service and maintenance, lack of future intent, and inability to meet growing aspirations, among others. Case studies of successfully implemented projects yield parameters that if taken into account, could lead to development of successful and sustainable projects.

\subsection{Renewable Energy Technologies in India: Progress and Challenges}

India, on account of its geographical position and terrain diversity is abundantly bestowed with multiple renewable energy resources including Solar, Wind, and Hydropower. India annually receives approximately 300 days of sunshine (5000 trillion kW of power) (Kapoor \& Dwivedi, 2017). Mani and Dhingra (2013) noted the advances made by India which is currently at the $5^{\text {th }}$ position globally in terms of installed wind energy capacity. Renewable energy projects in India may vary from large scale hydropower, wind, and solar projects to small, local/ village level off-grid, mini - grid and hybrid RET projects. During the $12^{\text {th }}$ Five Year Plan (2012-2017) the Ministry of New \& Renewable Energy (MNRE) proposed a target of $30000 \mathrm{MW}$ from all sources of renewable energy. As of December 2014, India's total renewable energy installed capacity was estimated at $33.8 \mathrm{GW}$, or an increase of $30 \mathrm{GW}$ or 1000 per cent from $3.9 \mathrm{GW}$ in 2002-03. Future progress in renewable capacity expansion is slated at more than 5 times the current scenario (from 32000 to $175000 \mathrm{MW}$ by 2022) (MNRE 2015). The breakup of this target is: $100 \mathrm{GW}$ Solar, $60 \mathrm{GW}$ Wind, $10 \mathrm{GW}$ Biomass and 5 GW Small Hydro (Lok Sabha 2015).

Research in context of India's renewable energy adoption has been focused on specific technologies and the issues and challenges. Raman (2012) detailed the technical components of a Solar Photovoltaic (PV) system and 
the techno - economic feasibility of solar PV systems for rural electrification in India. Madrigal and Porter (2013) presented the scenario of global wind energy capacity development situating India behind only China, United States, Germany, and Spain with 6.6 percent of worldwide coverage. Energy in India is a state subject and considerable policy and institutional shifts occur across states.

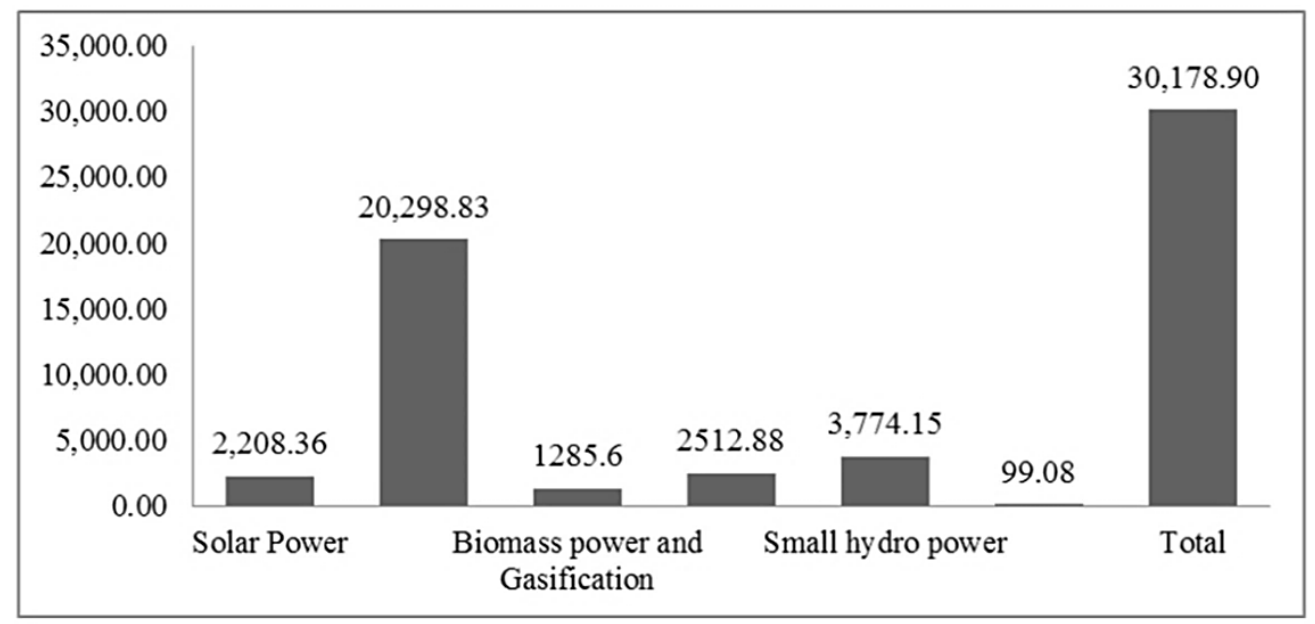

Figure 1. Grid Connected Renewable Energy in India (MW)

Source: Ministry of New \& Renewable Energy, January 31, 2014

With energy policies, regulations and outlook shifting from one state to the other, some states have capitalized on the available resources whereas other states have failed to do so. S. Pandey, et al., (2012) discussed determinants for success in promotion of solar energy in the state of Rajasthan, highlighting the significance of the policy, infrastructure, facilitation and governance aspects. Similarly, the progress in the state of Tamil Nadu in wind energy promotion and implementation has especially been considerable with 51 percent of installed capacities vis a vis the state's wind energy potential. George and Banerjee (2009) studied the impact of increasing wind power penetration on the dominant grid and projected a positive scenario for the state taking into account the annual load duration curve as a methodology for calculating peak load capacity and peak energy requirement. The study clearly illustrated the scope for RET projects to be dominant electricity providers for the state. Despite the evidence of slow uptake, India continues to have an incredible opportunity in the present space to edge forward in renewable energy technology application while providing energy to all its citizens. Further, considering grid expansion issues as detailed in the next section, off - grid energy access will be a prominent strategy for the nation.

\subsection{Off-grid or Decentralized RE Systems}

Grid Connected systems refer to those projects which are connected with electricity grid and supply power to the grid, while Off-Grid refers to those energy systems which have been designed for local use and not connected with electricity grid. Grid expansion is favoured by the beneficiaries for multiple reasons including the enabling policy and institutional framework, financing institutions, and finally, micro level household socio-economic position (Reddy \& Srinivas 2009). Other reasons related to favourability towards grid extension include reliability of power supply, fixed duration, and allowance for multiple usage purposes (TERI 2009), (cited in Palit \& Chaurey, 2011).

RETs such as solar photovoltaic (PV), biomass gasification and pico/ micro hydro- power are considered as alternative technologies in scenarios where central grid - based electricity supply is technically or economically infeasible or inadequate. Beck and Martinot (2004) defined distributed generation as electricity generation close to or on - site, and in need - based quantities, in comparison to large amounts generated in big sized centralized power plants. Palit and Chaurey (2011) construed off-grid energy systems as being inclusive of local mini - grid systems supplying a village or community, as well as standalone power supply systems for individual household usage (including solar home systems, individual solar lanterns, etc). Further, Kaundinya, Balachandra and Ravindranath, (2009) suggested two levels of decentralization: village level where meeting rural needs is the focus; and industry level, with focus on fulfilling industry demand and excess power handed over to the grid. Off-grid, or decentralized energy systems are favoured in context of remote location, grid infeasibility, and the 
ability to balance power needs through optimized usage of available renewable energy solutions (see Palit 2013). Avoided cost of transmission and distribution is an added advantage. Decentralized solutions also promote cost effective usage and self - reliance. Table 2 shows the status of off-grid RE systems deployment in India.

Table 2. Deployment of off-grid RE systems in India upto 31 March 2014

\begin{tabular}{ll}
\hline $\begin{array}{l}\text { Resource (Off-Grid/ Distributed Renewable Power } \\
\text { (including Captive/ Cogeneration Plants)) }\end{array}$ & Cumulative Achievements (in MW) \\
\hline Biomass Power / Cogeneration (non-bagasse) Plants & 531.80 \\
Biomass Gasifier Plants & 164.70 \\
Waste-to- Energy Projects & 132.70 \\
Solar Photovoltaic Power Plants & 174.40 \\
Aero-Generators/Hybrid Systems & 2.30 \\
Water Mills/ Micro Hydel Power Projects & 13.21 \\
\hline Total & $\mathbf{1 , 0 1 9 . 1 1}$ \\
\hline
\end{tabular}

Source: Comptroller and auditor general of India (CAG, 2015)

\section{Literature Review}

A number of studies have provided a comprehensive overview of renewable energy technologies and their uptake in India (see Bhattacharya and Jana, 2009; A., Kumar, K., Kumar, Kaushik, Sharma, \& Mishra, 2010). Khare, Nema, and Baredar (2013) presented an overview of solar and wind based renewable energy in India. Kapoor, Pandey, Jain, and Nandan, (2014) traced the evolution of solar energy policies and status in India across five year plans and challenges and barriers to its successful scaling up. Palit and Chaurey (2011) collated best practices across South Asia in context of off-grid rural electrification. International Finance Corporation (IFC 2012) compared market trends in context of Solar Off-Grid Lighting across India, Bangladesh, Nepal, Pakistan, Indonesia, Cambodia and Philippines. A comprehensive global review of renewable energy technologies and their global deployment scenario was given by Ellabban, Abu-Rub, and Blaabjerg, (2014).

Research around renewable energy technologies have focused on the policy and regulatory frameworks, and the technical feasibility and infrastructure constraints for large scale RET adoption (Geller, Schaeffer, Szklo, \& Tolmasquim, 2004; Schmid 2012, Sargsyan, Bhatia, Banerjee, \& Raghunathan, 2011). A number of studies have also focused on multiple types of barriers related to RET penetration. For instance Khare, Nema, and Baredar, (2013) analysed supportive policy instruments and regulatory, institutional, financial and technological barriers (drawn from IDFC, 2010). Eswarlal, Dey, and Shankar, (2011) highlighted 14 variables through Interpretive structural modeling for implementation of renewable energy, naming some as 'enablers' (see also Painuly (2001) for seven major barriers to RET penetration). Cust, Singh, and Neuhoff, (2007) studied multiple RET case studies to highlight techno - economic feasibility conditions, potential organizational models and consequent barriers influencing alignment of economics, institutional mechanisms, and participatory approach for large scale RET implementation at local level.

Literature in context of decentralized or off-grid electricity generation technologies has also been well-developed. A comprehensive review of decentralized approaches in Africa was presented by Tenenbaum, Chris, Tilak, and James (2014). World Bank (2008) provided a set of guidelines for decision making for design of sustainable off-grid rural electrification projects, including, but not limited to availability of technology choices, delivery mechanisms, financial and business model related dimensions, as also level of community involvement (see Board, 2008). A 2011 primer by Alliance for Rural Electrification concisely presented the technological, design, cost structures and business model characteristics of hybrid mini - grids (Rolland \& Glania, 2011). Kumar, Mohanty, Palit, and Chaurey, (2009) proposed a multi-step decision making tool for formulation of each stage of rural electrification projects (including both grid connected and off-grid projects). Bhattacharyya (2012) conducted a review of alternative Off-grid electrification options, including the sustainability dimension, recommending a hybrid approach.

Some examples from the ground where RET projects have been implemented at community level have shown potential while bringing forth challenges which need reflection in order for successfully scaling up. Hiremath, 
Kumar, Balachandra, Ravindranath, and Raghunandan, (2009) showcased the potentialities of off-grid RET contribution towards electricity production and consumption through case studies across possible technologies (bioenergy, solar, wind and hydro). On ground implementation case of RET for ensuring electricity access to remote villages in interior state of Rajasthan through off-grid renewables was shared by Chaurey, Ranganathan, and Mohanty, (2004). In another instance, Kanase-Patil, Saini, and Sharma, (2011) prepared an Integrated Renewable Energy Optimization Model (IREOM) for decentralized electrification of a cluster of 3 villages of Ranikhet reserved forest range of Uttarakhand state considering seasonal availability of resources and complementary demand profiles, at specific reliability values. Based on estimations of the average peak load of a remote rural household, Nouni, Mullick and Kandpal, (2008), recommended that villages with less than $50 \mathrm{~kW}$ peak load could be approached on priority for RET based decentralized electricity supply. A techno - economic feasibility analysis of two scenarios of rural village electrification (minimal energy access and productive energy access) through standalone rural village grids in Indonesia was carried out by Blum, Wakeling, and Schmidt, (2013). Sen and Bhattacharyya, (2014) proposed hybrid RET combination for fulfilling energy demand of a remote Chhattisgarh village using HOMER as an analytical tool.

Research on public perception of consumers \& stakeholders has also been well - documented (see Ruggiero, Onkila, \& Kuittinen, 2014; Walker, Wiersma, \& Bailey, 2014) In a study of a U.K. community RET project, the findings suggested reduction of psychological distance and integration of the project into daily lives of direct participants, with consequent increase in awareness, acceptance of renewables and low - carbon technologies, and increase in sustainable/ pro-environmental behavior (Rogers, Simmons, Convery, \& Weatherall 2012). Adams and Bell (2015) used an energy equity assessment tool to study two local energy generation projects and recommended application of perspectives based on equity and distributional justice at multiple phases of project planning and implementation.

Finally, recent research has focused on social, institutional and equity related concerns for decentralized local RET adoption. In recent years, advanced technologies have emerged around the creation of smart grid infrastructure, allowing energy and power sharing to shift from being a traditionally centralized, one way process from the generator and distributor to the customer, to a two way process that allows participation of the customer in generation, sharing, and benefiting from local power generation (see Sood, Fischer, Eklund, \& Brown 2009; Alvial-Palavicino, Garrido-Echeverría, Jiménez-Estévez, Reyes, \& Palma-Behnke 2011). Walker, Simcock, and Smith, (2012) studied the diverse range and meanings of Community Energy Systems in U.K. identifying their benefits, challenges and policy aspects. Newer approaches to decentralized electricity supply have dwelt on involvement of civil society, local municipal bodies, energy cooperatives, and prosumers (Bauwens 2013; Johnson \& Hall, 2014; Van Der Schoor, \& Scholtens, 2015).

\section{Emergence of Decentralized Renewable Energy Models}

Uptake of renewable energy technologies in large projects has so far been guided by subsidies, exemptions and large scale investments by the state or private investments. Mechanisms explored in this context have included Renewable Purchase Obligation (RPO), Renewable Energy Certificate (REC), Clean Development Mechanism (CDM), RGGVY scheme (household electrification and rural electricity infrastructure building through grid extension), Decentralized Distributed Generation Programme (DDG) where grid expansion is not feasible, Remote Village Electrification (RVE) Programme for areas not covered under DDG, and finally, Off-Grid and Decentralized Solar Application under Jawaharlal Nehru National Solar Mission (JNNSM). India's on ground implementation scenario for RET projects is at best mixed. Complexities surrounding the availability of land for large scale projects, frontloading of high capital investments, poor implementation of stated incentives and return options such as solar purchase obligations, renewable energy certificates, have weighed down the sector (see CAG, 2015). It is in this context that the relevance of decentralized, off - grid and mini grid projects becomes apparent.

With these and emerging stress on combating climate change through a low carbon economy perspective, efficient, demand - based, and distributed generation technologies have emerged as an alternative. The local, participative, and collective benefit nature of these systems of power generation have emphasized the need for public engagement and participation. Apart from strict techno - economic analysis of a potential project, inclusion of social, cultural, and behavioural factors are viewed as equally crucial to the success of a project. There is a need to study community oriented perspectives that crucially affect the ultimate success or failure of decentralized renewable energy technology implementation projects. As the case studies illustrate, private, public - private partnerships, \& community models are part of future approaches. 


\section{Methodology}

This paper focuses on the potential for development of decentralized, off - grid community models based on RETs in India. Accordingly, the paper tracks 9 case studies from different parts of India in the area of renewable energy technologies. For this purpose case studies have been drawn from Centre for Science and Environment's compendium of cases (CSE, 2014). The selection of case studies was influenced by the diversity of projects, innovations, areas of concern and learning and challenges presented by the case. A mix of government, private and NGO management cases were taken up to present across the sector issues.

Table 3. Case Studies analyzed in context of RET Application in India

\begin{tabular}{|c|c|c|c|c|c|c|}
\hline & & $\begin{array}{l}\text { Operational } \\
\text { Area }\end{array}$ & Capacity & Management & Pricing & Electricity output \\
\hline 1 & $\begin{array}{l}\text { Rajasthan } \\
\text { micro-grid solar } \\
\text { power - Gram } \\
\text { Power }\end{array}$ & $\begin{array}{l}\text { Neechli Babhan, } \\
\text { Pali district }\end{array}$ & $\begin{array}{l}5.5 \mathrm{kw} \text { for } 80 \\
\text { households }\end{array}$ & $\begin{array}{l}\text { Fee for service } \\
\text { model }\end{array}$ & $\begin{array}{l}\text { Rs. } 100 \text { recharge }=8 \text { watt } \\
\text { CFL for } 400 \text { hrs; Tariff }=\text { Rs. } \\
\text { 34/kwh; Cost Rs. } 25 \text { lakh. }\end{array}$ & $\begin{array}{l}4,600 \text { units generated from } \\
2012 \text { to Sept } 2013\end{array}$ \\
\hline 2 & $\begin{array}{l}\text { Assam SHS } \\
\text { system } \\
\text { experience }\end{array}$ & $\begin{array}{l}\text { Kutum village, } \\
\text { Sunitpur district }\end{array}$ & $\begin{array}{l}121 \text { households } \\
\text { SHS each }(2 \mathrm{cfls}, \\
1 \quad \text { battery, } \quad 1 \\
\text { charge controller })\end{array}$ & $\begin{array}{l}\text { Household, NGO } \\
\text { as intermediary }\end{array}$ & $\begin{array}{l}\text { Rs. } 13,476-90 \text { percent } \\
\text { MNRE subsidy; rest shared } \\
\text { between state govt. \& } \\
\text { household. } \\
\text { replacement after } 5 \text { years } \\
\text { (@3,500 Rs.) }\end{array}$ & $\begin{array}{l}\text { Saving of 300-400 Rs. Per } \\
\text { month on kerosene } \\
\text { expenses }\end{array}$ \\
\hline 3 & $\begin{array}{l}\text { Chhattisgarh } \\
\text { micro grid } \\
\text { experience }\end{array}$ & Deba village & $\begin{array}{l}4 \mathrm{kw} \text { solar power } \\
\text { plants (PV cells) } \\
\text { for } 75 \text { households }\end{array}$ & $\begin{array}{ll}\text { CREDA, } & \text { village } \\
\text { operator, } & \text { cluster } \\
\text { technician } & \end{array}$ & $\begin{array}{l}\text { Rs. } 25000 \text { per household (90 } \\
\text { percent MNRE subsidy) }\end{array}$ & $\begin{array}{l}28 \text { units of electricity per } \\
\text { day; } 7 \text { hours: } 4 \text { to } 6 \text { am } \\
\text { and } 6 \text { to } 11 \mathrm{pm}\end{array}$ \\
\hline 4 & $\begin{array}{l}\text { Solar - powered } \\
\text { Pumps in Bihar }\end{array}$ & $\begin{array}{l}34 \text { community } \\
\text { tube well pumps } \\
\text { in Nalanda } \\
\text { district }\end{array}$ & & $\begin{array}{l}\text { Private firm } \\
\text { maintenance along } \\
\text { with irrigation } \\
\text { department }\end{array}$ & $\begin{array}{l}\text { Rs. } 5 \text { per kattha ( } 125 \text { square } \\
\text { meter) per irrigation cycle; } \\
\text { unsubsidized } 3 \mathrm{HP} \text { solar } \\
\text { pump = Rs. } 4.5 \text { lakh) }\end{array}$ & \\
\hline 5 & $\begin{array}{l}\text { Odanthurai } \\
\text { Panchayat }\end{array}$ & $\begin{array}{l}\text { Odanthurai, } \\
\text { Coimbatore } \\
\text { District. Tamil } \\
\text { Nadu }\end{array}$ & $\begin{array}{l}350 \mathrm{kw} \text { wind } \\
\text { energy project }\end{array}$ & $\begin{array}{l}\text { Community: } \\
\text { panchayat bought } \\
\text { turbine from } \\
\text { Suzlon }\end{array}$ & 1.55 crores (loan) & $\begin{array}{l}\text { Panchayat self-electricity } \\
\text { need } 4.5 \text { lakh/ year. } 2.25 \\
\text { lakh unit sold to TNEB for } \\
\text { revenue of Rs. } 6.525 \text { lakh / } \\
\text { year }\end{array}$ \\
\hline 6 & $\begin{array}{l}\text { Micro - hydel } \\
\text { project, Odisha }\end{array}$ & $\begin{array}{l}\text { Putsil village, } \\
\text { Koraput district }\end{array}$ & $\begin{array}{l}14 \mathrm{Kw} \text { Micro } \\
\text { hydel project }\end{array}$ & NGO & $\begin{array}{l}\text { Rs. } 16,97,611 \text { - villagers } \\
\text { contributed } 12.5 \text { percent of } \\
\text { the project cost }\end{array}$ & $\begin{array}{l}3 \text { hours morning \& } 4 \\
\text { hours evening - } 60-70 \mathrm{~W} / \\
72 \text { households }\end{array}$ \\
\hline 7 & $\begin{array}{l}\text { Biogas } \\
\text { household } \\
\text { experiences in } \\
\text { Maharashtra }\end{array}$ & $\begin{array}{l}\text { Chittalwadi, } \\
\text { Akola, } \\
\text { Maharashtra }\end{array}$ & $\begin{array}{l}2 \text { cubic metre } \\
\text { (cum) digester } \\
\text { tank and above }\end{array}$ & Household & $\begin{array}{l}\text { Price varies depending upon } \\
\text { no. Of cattle and therefore } \\
\text { size of plant. Subsidy rs. } \\
8000 \text { for a } 2 \text { cum tank }\end{array}$ & $\begin{array}{l}\text { Cooking - for all domestic } \\
\text { fuel requirements need } 6 \\
\text { cum plant }\end{array}$ \\
\hline 8 & $\begin{array}{l}\text { Bihar biomass } \\
\text { experience }\end{array}$ & $\begin{array}{l}\text { Sahebganj } \\
\text { village } \\
\text { Muzaffarpur } \\
\text { district }\end{array}$ & $\begin{array}{l}\text { Two } 32 \mathrm{kw} \text { off } \\
\text { grid } \\
\text { gasification units }\end{array}$ & $\begin{array}{l}\text { Private. Husk } \\
\text { power systems }\end{array}$ & $\begin{array}{l}32 \text { kw plant@ Rs. } 18 \text { lakh. } \\
\text { Capital subsidy: Rs. 15,000 / } \\
\text { kw \& Rs. } 1 \text { lakh/ km upto } 3 \\
\text { km for transmission line }\end{array}$ & \\
\hline & Gujarat' biomass & $\begin{array}{l}\text { Example - } \\
\text { Kosamadi village } \\
\text { - Dang district }\end{array}$ & $\begin{array}{l}2 \text { cubic metre } \\
\text { (cum) biogas } \\
\text { plant }\end{array}$ & $\begin{array}{l}\text { Household, } \\
\text { cooperatives }\end{array}$ & $\begin{array}{l}\text { Rs. } 28000 \text { to } 30,000 \text {. MNRE } \\
\text { subsidy: Rs } 4000-1 \text { cum } \\
\text { plant/ } 8000 \text { Rs }-2 \text { to } 4 \text { cum } \\
\text { plant. Plus Rs. } 4000 \text { Gujarat } \\
\text { state government subsidy }\end{array}$ & $\begin{array}{l}\text { Cooking fuel - increasing } \\
\text { LPG prices }\end{array}$ \\
\hline
\end{tabular}

Selected RET typologies included solar, wind, micro - hydel, and biomass based projects. Table 4 showcases each case profile, pricing and management model and institutional support received. Through a synthesis of 
challenges, problems, learning's and innovations exemplified by each case study, the author arrives at a framework of evaluation that would be of assistance in understanding the issues and challenges in implementation. To build a resilient community model, specific lessons could be applied while designing such models. This paper proposes an evaluation framework which may be used across renewable energy technologies. The framework also proposes evaluation parameters specific to each of these five sectors.

\section{Drivers for Adoption of Renewable Energy Technology by Communities}

Table 4. Drivers of RET Application - Analysis of Case Studies drawn from CSE, 2014

\begin{tabular}{|c|c|c|c|c|c|}
\hline $\begin{array}{l}\text { Technical/ Design/ } \\
\text { Data/ } \\
\text { Infrastructure }\end{array}$ & $\begin{array}{l}\text { Social/ Ethical/ } \\
\text { Community }\end{array}$ & $\begin{array}{l}\text { Policy/ Governance/ } \\
\text { Regulatory }\end{array}$ & $\begin{array}{l}\text { Market/ } \\
\text { Model }\end{array}$ & nmental & $\begin{array}{l}\text { Financial/ } \\
\text { Economic } \\
\text { Sustenance }\end{array}$ \\
\hline $\begin{array}{l}\text { [5] Connection to } \\
\text { proximate grid }\end{array}$ & $\begin{array}{l}\text { Pre - commissioning } \\
\text { engagement }\end{array}$ & $\begin{array}{l}{[6] \quad \text { Chhattisgarh }} \\
\text { 3-tier system: O \& M }\end{array}$ & $\begin{array}{l}\text { [1] Fee for Service } \\
\text { Business model }\end{array}$ & $\begin{array}{l}{[5] \quad \text { Minimize }} \\
\text { ecosystem impact }\end{array}$ & 5] Seed Grant \\
\hline $\begin{array}{l}{[1,3] \quad \text { Operational }} \\
\text { Efficiency }\end{array}$ & $\begin{array}{l}\text { [6] Ownership via } \\
\text { sharing of funds/ } \\
\text { resources/ labour }\end{array}$ & $\begin{array}{lr}{[2] \quad \text { Theft/ }} & \text { sale of } \\
\text { subsidized } & \text { Solar } \\
\text { House } & \text { Systems } \\
\text { (SHS) } & \end{array}$ & $\begin{array}{l}\text { [9] Co-operative loan } \\
\text { model for biogas } \\
\text { plants }- \text { ensuring } \\
\text { capital, O \& M }\end{array}$ & $\begin{array}{l}\text { Safe distance from } \\
\text { forests and } \\
\text { habitations }\end{array}$ & $\begin{array}{l}\text { [5] Capital } \\
\text { Investment }\end{array}$ \\
\hline $\begin{array}{l}\text { Absence of local } \\
\text { testing facilities } \\
\text { leading to supply of } \\
\text { substandard } \\
\text { equipment [4] }\end{array}$ & $\begin{array}{l}\text { [3] Common } \\
\text { resources }- \text { health } \\
\text { centre/ tribal hostel/ } \\
\text { police camp driven } \\
\text { by micro - grid }\end{array}$ & $\begin{array}{l}\text { [2] Misconceptions } \\
\text { about RET } \\
\text { non-functioning due } \\
\text { to prevalence of } \\
\text { faulty equipment }\end{array}$ & $\begin{array}{l}\text { [8] Smaller scale, } \\
\text { improved designs for } \\
\text { biogas - alternate to } \\
\text { larger, grid - linked } \\
\text { plants with feed issue }\end{array}$ & $\begin{array}{l}\text { Favourable } \\
\text { hotspots } \\
\text { ecosystem } \\
\text { fragmentation }\end{array}$ & $\begin{array}{lr}\left.\begin{array}{lr}4, & 8\end{array}\right] \text { Raw } \\
\text { materials cost } \\
\text { increase- } \\
\text { shift }\end{array}$ \\
\hline $\begin{array}{l}\text { [1] Rigorous Data } \\
\text { requirements/ } \\
\text { Consequences from } \\
\text { absence of data }\end{array}$ & $\begin{array}{l}\text { [5] Exposure of } \\
\text { community leaders to } \\
\text { existing RET projects }\end{array}$ & $\begin{array}{l}\text { [3] Standardization } \\
\text { of O \& M system in } \\
\text { Chhattisgarh as } \\
\text { positive model }\end{array}$ & $\begin{array}{lr}\text { [8] Raw material } \\
\text { pricing } & \text { based on } \\
\text { biomass } & \text { calorific } \\
\text { value } & \end{array}$ & $\begin{array}{l}{[8] \quad \text { Consider }} \\
\text { avoidance of soil } \\
\text { nutrient loss from } \\
\text { straw burning }\end{array}$ & $\begin{array}{l}{[5] \quad \text { Sale of }} \\
\text { excess power to } \\
\text { nearest grid/ } \\
\text { customers }\end{array}$ \\
\hline $\begin{array}{l}\text { [6] Map usage \& } \\
\text { demand generation } \\
\text { timings }\end{array}$ & $\begin{array}{l}\text { [5] Role of advisors } \\
\text { in community hand } \\
\text { holding }\end{array}$ & $\begin{array}{l}\text { [2] Subsidy model - } \\
\text { substandard } \\
\text { equipment supply }\end{array}$ & $\begin{array}{l}\text { [8] Multiple buyers, } \\
\text { availability of } \\
\text { biomass feed supply }\end{array}$ & $\begin{array}{lr}{[6] \quad \text { Ensuring }} \\
\text { Ecological } & \text { Flow } \\
\text { availability } & \end{array}$ & $\begin{array}{l}\text { [4] Smart } \\
\text { Metering - avoid } \\
\text { power theft }\end{array}$ \\
\hline $\begin{array}{l}\text { [6] Minimization of } \\
\text { Dump Loss }\end{array}$ & $\begin{array}{l}\text { Involvement/ training } \\
\text { in operation and } \\
\text { maintenance }\end{array}$ & $\begin{array}{l}\text { State provision of } \\
\text { viability support - } \\
\text { thin margins }\end{array}$ & & $\begin{array}{l}{[8] \text { Impact of }} \\
\text { combustion/ } \\
\text { Biomethanation }\end{array}$ & $\begin{array}{l}\text { Demand } \\
\text { forecasting for } \\
\text { longer period }\end{array}$ \\
\hline $\begin{array}{l}\text { [5] Inadequate } \\
\text { transmission } \\
\text { infrastructure }\end{array}$ & $\begin{array}{l}\text { [6] Excess power for } \\
\text { income generation } \\
\text { activities }\end{array}$ & $\begin{array}{l}\text { [8] Regulation of } \\
\text { private market } \\
\text { system }\end{array}$ & $\begin{array}{ll}\text { [2] Local business } \\
\text { skills/ } & \text { capacity } \\
\text { building } & \end{array}$ & $\begin{array}{l}\text { Impact of linear } \\
\text { intrusion }\end{array}$ & $\begin{array}{l}{[2,5,6,7,8,9]} \\
\text { Loans/ subsidies/ } \\
\text { exemptions }\end{array}$ \\
\hline $\begin{array}{l}\text { [5] Identification of } \\
\text { Favourable Hotspots }\end{array}$ & $\begin{array}{l}\text { [5] Committee/ group } \\
\text { formation }\end{array}$ & & & $\begin{array}{l}\text { Compliance to EIA } \\
\text { Procedures }\end{array}$ & $\begin{array}{l}{[3] \quad \text { Cost }} \\
\text { Community }\end{array}$ \\
\hline $\begin{array}{l}\text { [4] Hybrid models } \\
\text { for } \quad \text { improved/ } \\
\text { assured } \\
\text { Managing } \\
\text { variability }\end{array}$ & $\begin{array}{l}\text { [6] Match timing of } \\
\text { power generation/ vs. } \\
\text { village chores/ } \\
\text { activities }\end{array}$ & $\begin{array}{l}\text { Excise duty } \\
\text { exemption and tax } \\
\text { holidays to support } \\
\text { long gestation project } \\
\text { period }\end{array}$ & $\begin{array}{l}{[8] \text { Employment }} \\
\text { generation as a } \\
\text { complementary } \\
\text { strategy in biomass }\end{array}$ & $\begin{array}{l}\text { sustainable } \\
\text { sanitation } \\
\text { positive impact }\end{array}$ & $\begin{array}{l}{[6] \quad \text { Extensive }} \\
\text { evaluation by } \\
\text { Financial } \\
\text { Institutions }\end{array}$ \\
\hline $\begin{array}{l}\text { Potential to scale/ } \\
\text { risk } \\
\text { re-engineering }\end{array}$ & $\begin{array}{l}\text { [4] Solar irrigation } \\
\text { pumps - prevent over } \\
\text { irrigation, water table } \\
\text { issues }\end{array}$ & $\begin{array}{l}\text { [3] Lax monitoring \& } \\
\text { poor service due to } \\
90 \text { percent subsidy } \\
\text { model }\end{array}$ & & $\begin{array}{l}\text { Reduction of } \\
\text { quantum of waste } \\
\text { through } \\
\text { co-generation }\end{array}$ & $\begin{array}{l}{\left[\begin{array}{l}2, \\
\text { Community }\end{array}\right]} \\
\text { corpus fund/ } \\
\text { Economic group }\end{array}$ \\
\hline $\begin{array}{l}\text { Mimic river's } \\
\text { natural flow as } \\
\text { design parameter }\end{array}$ & $\begin{array}{l}{[6] \quad \text { Power for }} \\
\text { education, play, } \\
\text { house } \quad \text { work, } \\
\text { community activity }\end{array}$ & $\begin{array}{l}\text { [4] Uncertain policy } \\
\text { regime } \\
\text { development of dead } \\
\text { infrastructure }\end{array}$ & & $\begin{array}{l}\text { [6] Principle of } \\
\text { minimal damage }\end{array}$ & $\begin{array}{l}\text { Dedicated funds } \\
\text { for risk/ failure } \\
\text { assessment }\end{array}$ \\
\hline $\begin{array}{l}\text { Minimize mid } \\
\text { project } \quad \text { shifts } \\
\text { through rigorous pre } \\
\text { - assessments }\end{array}$ & $\begin{array}{l}\text { [5] Cross learning - } \\
\text { proximity to other } \\
\text { decentralized grid/ } \\
\text { community models }\end{array}$ & $\begin{array}{l}\text { [3] Issues related to } \\
\text { on-paper } \\
\text { electrification/ } \\
\text { de-electrified villages }\end{array}$ & & $\begin{array}{l}\text { Impact of multiple } \\
\text { Small Hydro Power } \\
\text { projects on river }\end{array}$ & $\begin{array}{l}{\left[\begin{array}{ll}5, & 6\end{array}\right] \text { Income }} \\
\text { generation } \\
\text { activities from } \\
\text { excess supply }\end{array}$ \\
\hline
\end{tabular}




\begin{tabular}{|c|c|c|c|}
\hline $\begin{array}{l}{[7,9] \text { Small scale, }} \\
\text { improved biomass } \\
\text { models }\end{array}$ & $\begin{array}{ll}\text { [6] Capacity building } & \text { [9] Withdrawal of } \\
\text { for village } & \text { subsidies impacted } \\
\text { representatives } & \text { uptake of biogas }\end{array}$ & $\begin{array}{l}\text { Mimicking river's } \\
\text { natural flow }\end{array}$ & $\begin{array}{l}\text { [1] Availability } \\
\text { of reliable data }\end{array}$ \\
\hline $\begin{array}{l}\text { [1] High precision } \\
\text { manufacturing }\end{array}$ & $\begin{array}{ll}\text { Incentives } & \text { for } \\
\text { decentralization } & \end{array}$ & Impact of leachates & $\begin{array}{l}\text { Earnings after } \\
\text { loan repayment }\end{array}$ \\
\hline$[1,5,6] \quad$ Energy/ & Innovative models & & [1] Customers' \\
\hline Battery Banking & for resource sharing & & $\begin{array}{l}\text { financial } \\
\text { viability }\end{array}$ \\
\hline $\begin{array}{l}\text { [9] Lack of spares, } \\
\text { technicians }\end{array}$ & $\begin{array}{l}\text { [6] Consider growing } \\
\text { aspirations }\end{array}$ & & $\begin{array}{ll}\text { Site } & \text { selection } \\
\text { issues } & \end{array}$ \\
\hline $\begin{array}{l}{[9] \quad \text { Indigenous }} \\
\text { Technologies }\end{array}$ & $\begin{array}{l}\text { Sociocultural issues } \\
\text { in site selection }\end{array}$ & & \\
\hline $\begin{array}{l}\text { [5] Power wheeling } \\
\text { mechanism }\end{array}$ & & & \\
\hline
\end{tabular}

The analysis of case studies shows a number of factors that were altogether responsible for the success of each project. The factors were further synthesized into higher order factors. The higher order factors include technical/ design/ data and infrastructure; financial/ economic considerations, policy \& regulatory, environmental aspects, market/ business model and social/ ethical/ community linked aspects. The analysis illustrated that in each phase of the project, whether pre - commissioning, construction \& installation phase, or post installation, the need for an enabling economic environment and technical guidance to the community was clearly visible. Environmental considerations also emerged as a crucial area, illustrating that simply using a renewable technology does not ensure that the project necessarily meets the prevalent environmental obligations and secure ecosystem services in the medium and long term period. Equally significant was the range of community linked parameters and importance of considering individual and local aspirations, culture, and context, both during the design as also post implementation phases. Cross - cutting factors included availability of data, site selection parameters, productive energy use possibilities, and capacity building throughout the value chain - village representatives, local youth/ entrepreneurs, technicians, and supervisors. Emphasis was on long - term perspective, inculcating a system based approach, inclusion of design parameters such as adequate and rigorous data, appropriate site selection, and adopting a community centered approach.

\subsection{Financial/ Economic Considerations}

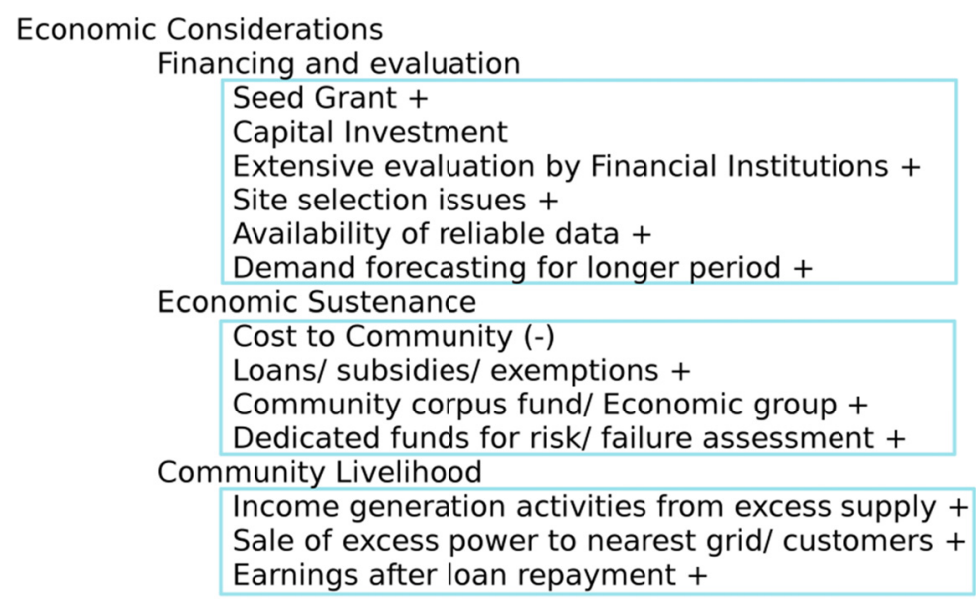

Figure 2. Economic Considerations: Factors for RET implementation

Economic considerations include the financing and evaluation linked parameters, i.e. availability of seed grant or capital investment to cover the initial construction/ implementation costs; the positive influence of extensive project evaluation done by financial institutions/ banks; positive impact of careful site selection; and the availability of rigorous, localized, technical data as a crucial requirement, esp. for solar, wind, and hydro power technologies. 
Examination of cases indicates that sustainable examples occur when communities have been oriented and prepared to manage recurring equipment related issues, for instance, in solar home systems, the need to replace batteries after a specific period ( 5 years). The current project design stipulates that replacement costs are to be borne by the community or individual households, for which the beneficiaries do not receive any funds, grants or subsidies. Instances where communities have set up corpus fund for financing these replacements are therefore a positive marker towards long term sustainability of the project (e.g. Assam's off - grid solar projects). Similarly in the pre-commissioning stage, sustainable projects are more likely to have been rigorously evaluated for their financial viability. Another aspect that is very crucial for community based models is productive energy generation i.e., the potential to carry out income generation activities, whether through sale of excess power to other buyers, or setting up of community based activities such as rice mills, or other small household based activities (see instance of Micro-hydel based community project in Odisha). Additional benefits here include rekindled community interest and higher probability of sustained system use.

\subsection{Technology and Infrastructure}

The categories falling under technology and infrastructure related drivers include ensuriing operational efficiencies, and the ability to fulfill demand during the lean season (lean season for solar plants/ systems would be the winter season when foggy conditions lead to lower output, similarly, in case of hydro power, the dry and winter seasons would be leaner periods). Hybrid models where multiple power sources are combined were found to improve efficiency, output, and therefore had improved community percpetions. System based approaches were found to be successful through completion of the loop between community owners vs. other customers, and/or the nearest grid; as well as ensuring power banking and energy storage, and also minimzation of dump load through matching energy demand and supply.

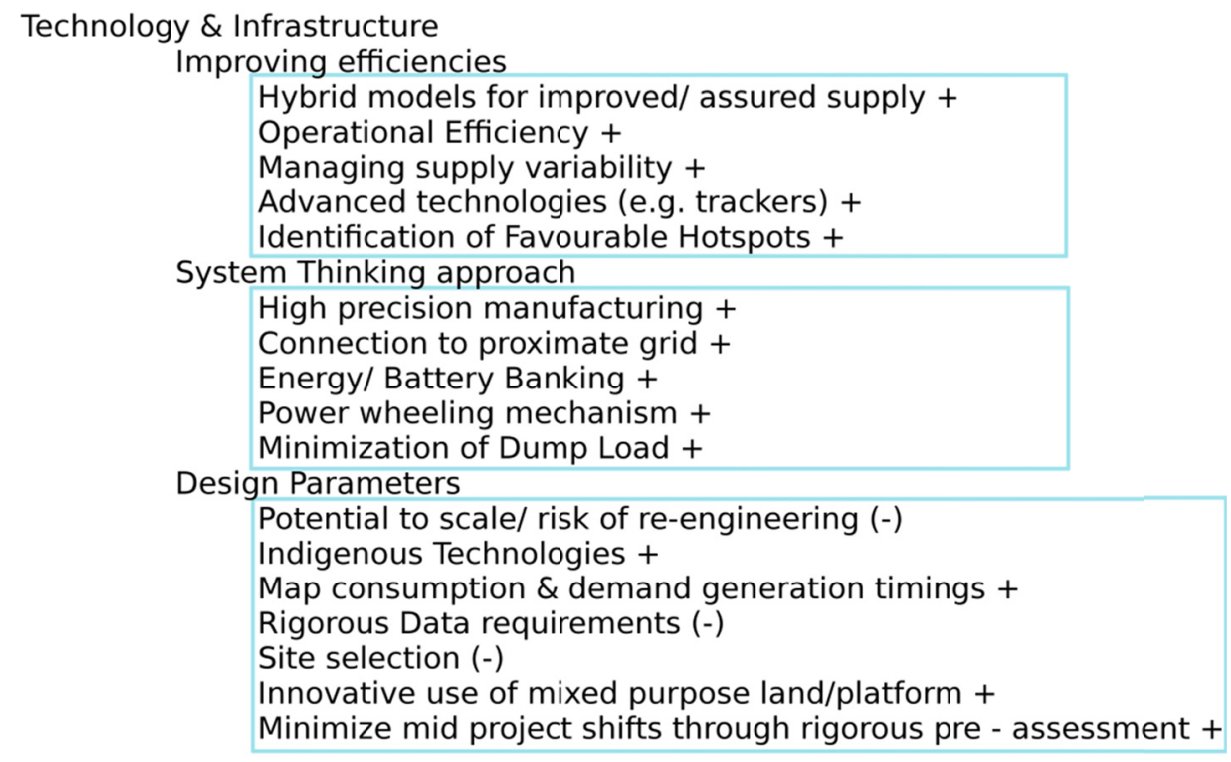

Figure 3. Technical, Design \& Infrastructure: Factors for RET implementation

Similarly, design parameters include customization capacities for specific community requirements such as seasonal demands related to irrigation use; or power utilization for household chores at specific times of the day depending upon the agricultural/ seasonal requirements. This may extend project usability and public perception, particularly in case of projects sans power banking option. Engaging the community in solving issues related to timing of power supply and demand generation is thus a positive marker. Similarly, significance of site selection and rigorous scientific data are seen as crucial in case of both wind and solar energy based projects for robust design \& hence need emphasis at the project commissioning stage itself. Due to lack of reliable data, many projects fail to get commissioned on the ground. Alternatively, design changes during the project implementation phase may be extremely difficult and cost intensive. Lack of data or proper site selection may consequently lead to mid project reassessments or shifts and not yield optimum leverage in cases where scaling up is necessitated for generational capacity increase. Another equally significant parameter is that of identifying favourable hotspots linked to wind, hydropower and solar energy technologies. Locations with maximized wind velocity availability 
for large portions of the year are beneficial; direct incident radiation availability is a key parameter in case of solar projects; and similarly, maximized potential and kinetic energy for small hydro power projects can alter the project success ratios considerably.

\subsection{Policy \& Regulatory Issues}

One unanticipated consequence of the Ministry of New and Renewable Energy (MNRE)'s subsidy provisions in solar off-grid systems (note 3 ) is the lack of accountability and long term interest by official functionaries as well as recipients. This has led to supply of substandard equipment that becomes dysfunctional within months, or needs constant repair. Instances on the ground have shown that lack of ownership results in grey market sale of the subsidized systems, as well as corruption (such as on - paper installation) etc (see CAG, 2015). The lack of interest in the long term sustainability of the project is also evident in the state response, since it is the states that are responsible for third party monitoring and operation and maintenance (see Borah, R.R., Palit D., \& Mahapatra S., (2014)). In this disappointing scenario, the example of state of Chhattisgarh, which has implemented a very successful solar mini - grid programme deserves special mention. The specific actions undertaken by the government include standardization of the operation and maintenance system, with a 3 tier monitoring and service model in place (CSE, 2014), common resources such as schools, tribal hostels, police camps etc. serving as the point of location for the mini grid among others, and, planning in advance about growing aspirations and demands from the community to remain relevant and avoid conversion to conventional fuels such as diesel powered generators/ LPG. Another aspect that is relevant from the policy and regulatory aspect refers to the degree of regulation for the private market, whether it be equipment providers, suppliers of irrigation pumps, or fee - for service models. There is increasing recognition that the regulatory agencies need to be aware of the avenues for misrepresentation and faulty supplies in grey markets. Regulators need to be cautious about allowing profitability of private players while reassuring customers, preventing shift to conventional fuels on account of malpractice, substandard or faulty equipment, or other similar actions.

\subsection{Market/ Business Model Related Parameters}

In the recent years, a number of business models have evolved in the renewable energy sector. Ranging from fee for - service models, cooperatives, public - private - partnerships, and private enterprises, the Indian market is well on the way to maturing (See GNESD, 2014 for a close look at various community based models for mini-grids in India). Essential strategies for the sector include capacity building of local entrepreneurs and providing support for initial capital investments, viability gap funding, improving margins, backup in case of system shortfalls and adequate infrastructure. Successful projects were identified as those that had given adequate thought to training and capacity building of entrepreneurs, mechanics, and supervisors in the sector. Evolution of business models that favour both the developer and the customer retain a basic advantage in terms of convenience, budgeting, and preventing the free lunch attitude (e.g., case of gram power in Rajasthan with pre-paid electricity credits and smart meters to prevent power thefts). Communities have also acknowledged the overall cost reduction advantage brought by RET based models even though they may prefer hybrid models (complement renewable energy with other conventional modes such as kerosene, or diesel generators), for instance, in case of irrigation (e.g., solar water pumps, and the experience of Chhatisgarh). Managing supply variability was found to be crucial especially for projects based on biomass, with competing industries (e.g. cement and brick kilns, packaging industry) willing to pay equal or higher amounts for the biomass feeds (e.g. rice husk based biomass plants in Chhatisgarh). Added services and customer friendly practices were found to be positively linked, for instance, to biogas implementation (instance of cooperatives in Gujarat where profits from milk supplied by cattle owners could be deducted as loan instalments for their biogas plants. Additionally, the cooperative assisted through construction material, and providing free spare parts and access to technicians).

\subsection{Environmental Considerations}

Environmental issues also play a prominent role in defining the long-term trajectory of the project. Each renewable energy technology sector, be it solar, wind, small hydro, or biomass, has a positive or negative association with the environment. A key element here is land - acquiring land for big infrastructural projects, whether solar, wind, or hydro, may impinge upon forest land and cause ecosystem fragmentation. Similarly, a specific principle related to biodiversity conservation is that of safe distance from forests and habitations. Wind power projects create noise, flicker, and electrocution related hazards for human beings and animals. At the same time, project developers need to take into account the requirement of operationalizing projects at desirable hotspots for maximizing power generation. Projects that are able to successfully overcome the trade - offs between these two have improved potential for success. Environmental considerations are particularly high in significance for small hydro power and the life of a river. Building multiple Small Hydro Power projects over a single river, if not carefully designed to 
ensure ecological flow (e-flow) or a river's capacity to flow over leaner periods (winter, dry summer months) may lead to multiple concerns. Centre for Science and Environment, 2014 recommended in context of e-flow to have the criteria of 30 per cent of water flow in warmer season (May to Oct) \& 50 per cent in cold (Nov to Apr) to be left aside at the project design stage itself.

Environmental Considerations
Biodiversity Conservation
\[ \begin{array}{l}\text { Minimize forest diversion }+ \\ \text { Safe distance from forests and habitations }+ \\ \text { Favourable hotspots vs. ecosystem fragmentation }+ \\ \text { Maintain ecosystem services } \\ \text { Mimicking river's natural flow }+ \\ \text { Ensuring Ecological Flow availability }+ \\ \text { Principle of minimal damage }+ \\ \text { Impact of linear intrusion (-) } \\ \text { Impact of multiple SHPs on river (-) } \\ \text { Compliance to ElA Procedures } \\ \text { Community health and well - being } \\ \text { Impact from combustion/ Biomethanation (-) } \\ \text { Impact of leachates (-) } \\ \text { Reduction of quantum of waste through co-generation }+ \\ \text { Complementary action of sustainable sanitation }+\end{array} \]

Figure 4. Environmental Aspects: Factors for RET implementation

In the state of Uttarakhand, river based power projects have come into scrutiny over their role in the 2013 flash floods. Environmental considerations also include the overall health and well - being of community. In general, renewable energy is considered environment friendly, and is often devoid of carbon and other emissions. For instance, replacing diesel powered irrigation pumps with solar pumps, apart from being financially viable, avoids year on year carbon emissions to a large extent. However, this is not true in the case of biomass and waste - to energy projects, where incomplete combustion is utilized as one of the methods for energy generation.

\subsection{Social/ Community Related Parameters}

Renewable Energy Technologies (RETs) have a long - term and continuous interface with the communities where they may be located, irrespective of technology type and scale. Community specific parameters thus retain a defining role in the overarching potential of RETs. Expansion of RET projects on a massive scale, therefore, can only be possible if these stakeholders, their aspirations, their needs, and their conflicts are considered at each phase of project design, commissioning, construction, maintenance, and possible expansion. Involvement of the community at the pre-commissioning stage when financial and evaluation assessments are being carried out is thus one of the key recommendations. Ensuring the community's involvement in site identification and selection, and avoidance of socio-cultural, economical conflicts significantly impact the overall project. Further, pre commissioning engagement would also assist the proposers in understanding the daily needs of the community and devising the proper mix of renewable energy. These subsequently promote community ownership of the project. Exposing community leaders to existing renewable technologies, cross learning from other working community models, and capacity building promotes local decision making, and accountability for long - term operational and maintenance issues (see instances from community entrepreneurship in wind farms in Tamil Nadu, micro - hydel in Odisha, and bio-gas in Gujarat).

A specific community related factor refers to the technical guidance provided to communities. The role of advisors, NGOs, and government functionaries in community hand - holding was found to be extremely useful (for instance, cases from Rajasthan, Assam). In Odisha, for instance, excess power was utilized for community specific income generation activities dovetailed with the timing of power generation. 


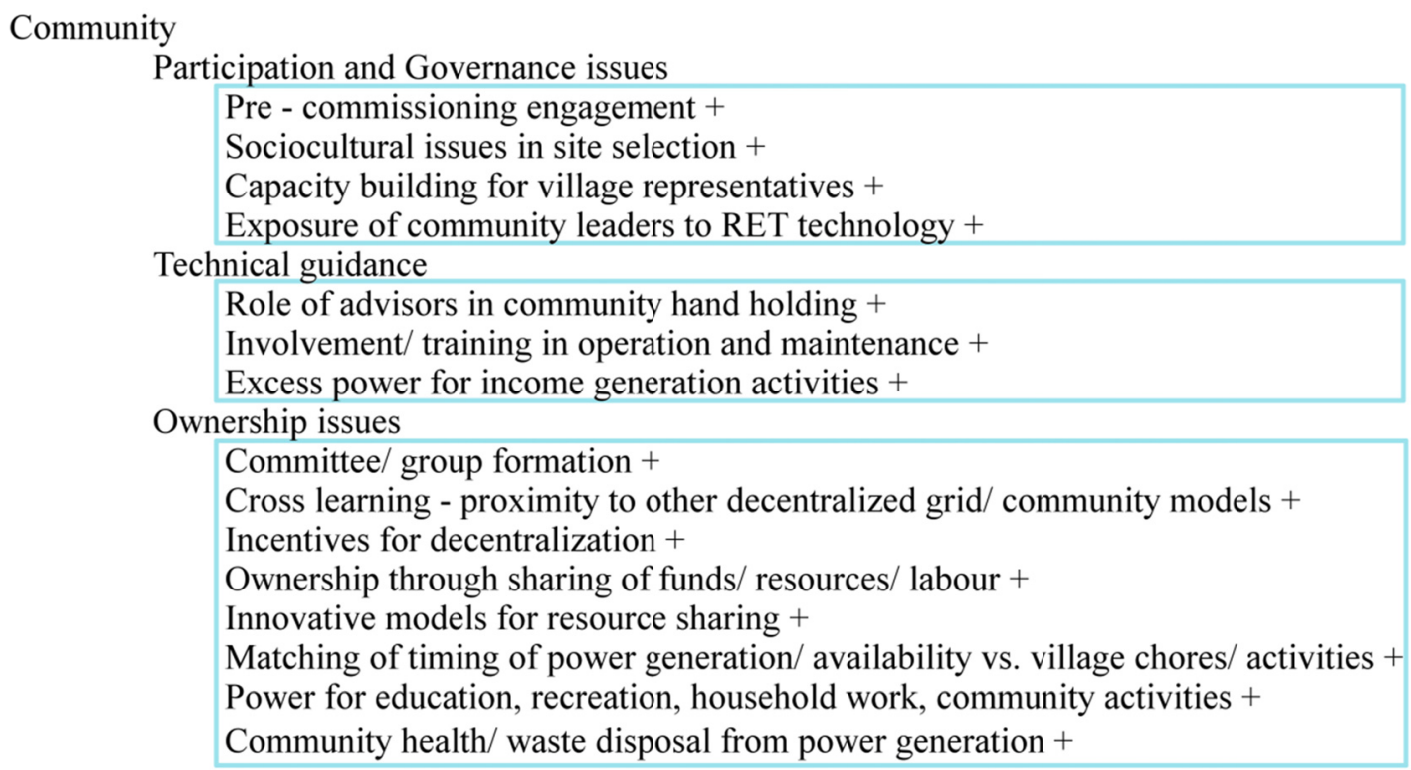

Figure 5. Social \& Community related Parameters: Factors for RET implementation

Once community ownership of the project is ensured, a comprehensive range of actions could be undertaken towards long - term viability and sustenance of the project. These include community group formation, training of individuals for technical repair and maintenance, community corpus for risks/ potential failures or generational expansion, innovative models for resource sharing, optimizing power capacity usage for diverse beneficiaries (school students, health centre, community centre activities, shared TV set for the community, community owned solar powered irrigation pumps, rice mills, household goods shops) and so on. Communities may also contribute materials, funds, or labour for the construction/ maintenance/ service of the project. It may also lead the community to work out models for profit development over the life time of the project and engage as power providers to nearby areas through power banking, wheeling mechanism, or utilizing their proximity to nearby grid facility. One crucial aspect of community engagement is building their understanding of the significance of RETs over conventional models of power generation such as firewood, kerosene, diesel generators, and so on. As grid expansion takes place through natural growth model, it is this sense of ownership, responsibility, and pride that will allow communties to either remain off - grid, \& even choose to earn funds through turning producers and adding to grid capacities. This would only be possible when consumers understand the benefits and requirements as a country, to continue on the renewable energy track as an option, rather than a constraint, or only viable in absence of grid availability.

\section{Proposing a Framework of Evaluation}

A number of studies have discussed approaches to off-grid energy development in India. Mishra and Sarangi (2011) presented a sustainability framework based on decision hierarchy for off-grid energy services. The World Bank (2008) published an operational guideline for designing sustainable off-grid rural electrification projects. Kumar, Mohanty, Palit, and Chaurey, (2009) attempted to standardize planning and formulation of off-grid projects through a comprehensive technical decision making tool. The present work builds on these technical tools to incorporate socio - technical drivers through presentation of an evaluative framework. The Framework of evaluation, as illustrated in the figure above, enumerates the key drivers related to sustainable community based RET projects. 


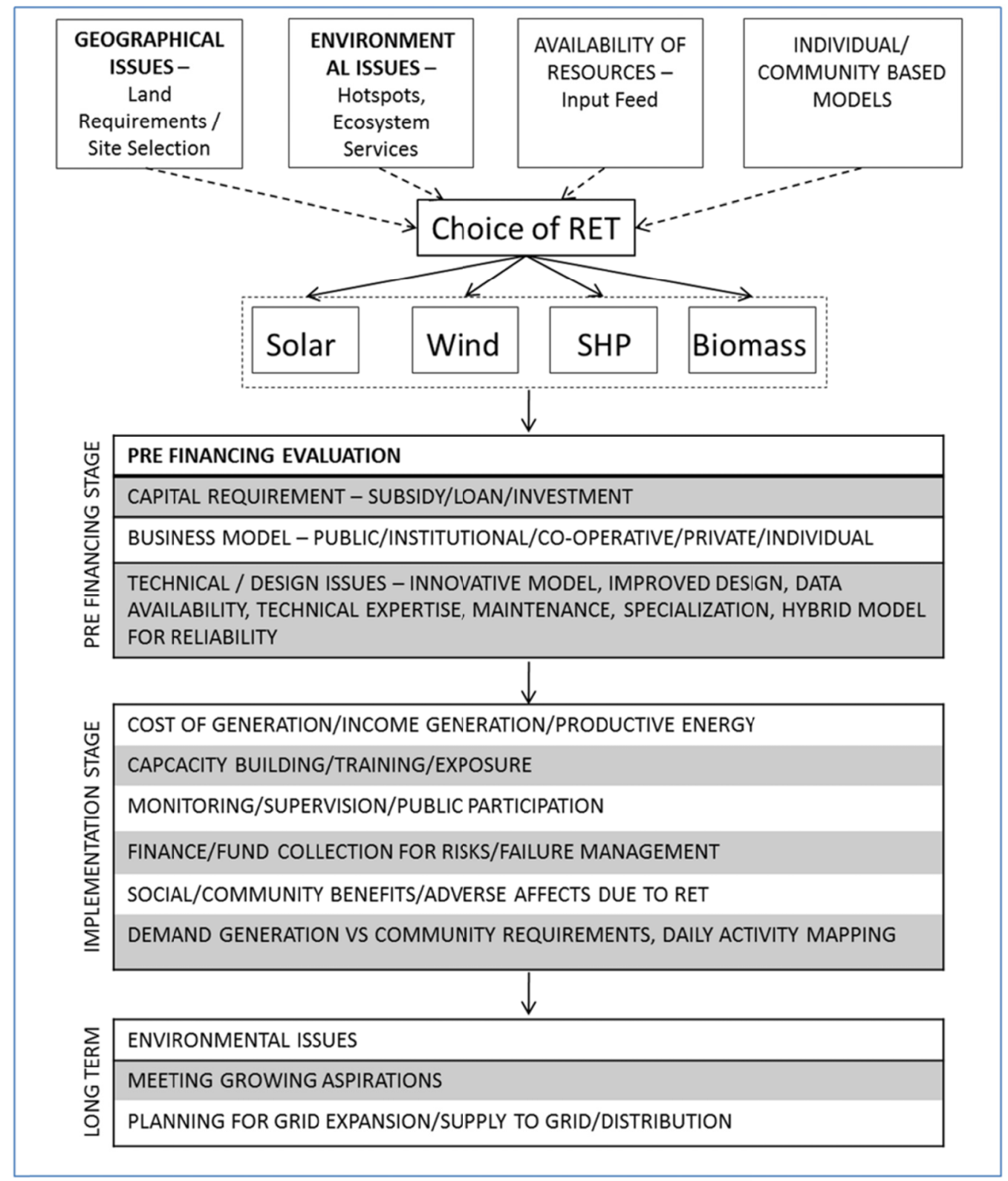

Figure 6. Framework of Evaluation: Community Applications of RETs

This evaluation framework presupposes the identification of renewable technology type on the basis of availability of renewable resource (such as wind, micro-hydel, agricultural or woody biomass) in adequate quantities and over the project timeline. Inclusion of environmental issues such as hotspots, ecosystem services, defragmentation, maintaining ecological flow levels (for small hydro), also need to be taken into consideration at the pre-commissioning stage. Site selection issues need to be broad - based to include social, cultural and environmental issues and not just techno - economic feasibility concerns. Finally, ensuring all social and cultural conflicts have been addressed and community perceptions aligned to the project are essential at the project identification stage itself.

The framework further elaborates on the areas of concern that might be evident at each stage of the project commissioning and implementation. At the pre-financing stage, for instance, technical and financial concerns are predominant for the project viability. Once the project is commissioned, other factors dominate the conversation. These include potential of productive energy usage, training and optimization, monitoring and community 
involvement, managing social concerns, and planning for future needs. In the long-term, ensuring minimal environmental damage while meeting increasing energy demands, and choices for grid engagement need addressing. Providing a holistic overview at the planning stage itself allows state functionaries, private developers, and local beneficiaries to take a whole system approach, and actively debate long-term issues in addition to the immediate concerns of project siting, scale, and economics typically under consideration.

\section{Recommendations and Conclusion}

Renewable Energy Technologies portend a very different future for India if accepted as a mainstream option. Careful design of schemes, incentives, and exploiting technological advancements to maximize power generation potential has laid the ground for the sector in India. However, at best, the utilization rates across sectors remain at $20-60$ per cent of the capacities even in best performing states in India, with most states yet to take benefits from the sector. Ensuring wide stakeholder involvement esp. at community level, and careful customization of projects is a necessity.

Examples from case studies have illustrated both concerns and pitfalls, and positive deviance from the norm. Designing of community models with participation, technical inputs, training and capacity building, and long term economic gain has the potential to reemphasize RETs as a way forward for universal energy access and security, as well as income generation through community livelihoods. Four recommendations can be put forth based on the framework of community models of RET in Indian Context namely:

1) Skill development in RETs can be one major revenue stream for rural youth, who can also be encouraged to turn renewable energy entrepreneurs and provide at - scale models for household/ community and special institutional requirements.

2) As technological advancements hold considerable promise for further diminishing the gap between conventional coal powered generation, the need to invest in R \& D needs encouragement. Special SEZ's for RET technology may be considered as high precision manufacturing will remain to be a crucial parameter.

3) Research \& Development Centres supporting rigorous and real - time RET data collection and analysis along the lines of meteorological data collection is also one specific area that needs consideration of policy makers.

4) Standardization of models and operating procedures in the case of community models is the need of the hour and states have shown the way forward (instance of Chhattisgarh for off - grid solar, including maintenance and service system architecture)

\section{Acknowledgments}

The author would like to expressly acknowledge the Centre for Science and Environment, New Delhi a policy think tank in the field of environment and sustainable development. Their comprehensive publication: State of Renewable Energy in India: A Citizen's Report provided the source for the analytical case studies utilized in the above work.

\section{References}

Adams, C. A., \& Bell, S. (2015). Local energy generation projects: assessing equity and risks. Local Environment, 20(12), 1473-1488. http://dx.doi.org/10.1080/13549839.2014.909797

Alvial-Palavicino, C., Garrido-Echeverría, N., Jiménez-Estévez, G., Reyes, L., \& Palma-Behnke, R. (2011). A methodology for community engagement in the introduction of renewable based smart microgrid. Energy for Sustainable Development, 15(3), 314-323. http://dx.doi.org/10.1016/j.esd.2011.06.007

Bauwens, T. (2013). What roles for energy cooperatives in the diffusion of distributed generation technologies? Centre, for Social Economy, University of Liege, Belgique.

Beck, F., \& Martinot, E. (2004). Renewable energy policies and barriers. Encyclopedia of energy, 5(7), 365-383.

Bhattacharya, S. C., \& Jana, C. (2009). Renewable energy in India: historical developments and prospects. Energy, 34(8), 981-991. http://doi.org/10.1016/j.energy.2008.10.017

Bhattacharyya, S. C. (2012). Review of alternative methodologies for analysing off-grid electricity supply. Renewable and Sustainable Energy Reviews, 16(1), 677-694. http://doi.org/10.1016/j.rser.2011.08.033

Bhattacharyya, S. C. (2015). Influence of India's transformation on residential energy demand. Applied Energy, 143, 228-237. http://doi.org/10.1016/j.apenergy.2015.01.048

Bhattacharyya, S. C., Palit, D., \& Kishore, V. V. N. (2014). Suite of off-grid options in South Asia. In Mini-Grids for Rural Electrification of Developing Countries (pp. 11-36). Springer International Publishing. 
http://doi.org/10.1007/978-3-319-04816-1_2

Blum, N. U., Wakeling, R. S., \& Schmidt, T. S. (2013). Rural electrification through village grids-Assessing the cost competitiveness of isolated renewable energy technologies in Indonesia. Renewable and Sustainable Energy Reviews, 22, 482-496. http://doi.org/10.1016/j.rser.2013.01.049

Board, M. S. (2008). Designing Sustainable Off-Grid Rural Electrification Projects: Principles and Practices. Retrieved from http://siteresources.worldbank.org/EXTENERGY2/Resources/OffgridGuidelines.pdf

Borah, R. R., Palit, D., \& Mahapatra, S. (2014). Comparative analysis of solar photovoltaic lighting systems in India. Energy Procedia, 54, 680-689. https://doi.org/10.1016/j.egypro.2014.07.309

CAG. (2015). Report No. 34 of 2015 - Performance Audit on Renewable Energy Sector in India Union Government, Ministry of New and Renewable Energy. Retrieved from http://www.cag.gov.in/content/report-no-34-2015-performance-audit-renewable-energy-sector-india-uniongovernment-ministry

CEA. (2016). Monthly Report on Installed Capacities. Retrieved from http://www.cea.nic.in/reports/monthly/installedcapacity/2016/installed_capacity-02.pdf

Centre for Science and Environment. (2014). State of Renewable Energy in India: A Citizen's Report.

Chaurey, A., Ranganathan, M., \& Mohanty, P. (2004). Electricity access for geographically disadvantaged rural communities - technology and policy insights. Energy policy, 32(15), 1693-1705. http://doi.org/10.1016/S0301-4215(03)00160-5

Cust, J., Singh, A., \& Neuhoff, K. (2007). Rural Electrification in India: Economic and Institutional aspects of Renewables (No. 0763). Faculty of Economics, University of Cambridge. Retrieved from https://ssrn.com/abstract $=2760810$

Ellabban, O., Abu-Rub, H., \& Blaabjerg, F. (2014). Renewable energy resources: Current status, future prospects and their enabling technology. Renewable and Sustainable Energy Reviews, 39, 748-764. http://doi.org/10.1016/j.rser.2014.07.113

Eswarlal, V. K., Dey, P., \& Shankar, R. (2011). Enhanced renewable energy adoption for sustainable development in India: Interpretive structural modeling approach. http://dx.doi.org/10.3384/ecp11057351

Geller, H., Schaeffer, R., Szklo, A., \& Tolmasquim, M. (2004). Policies for advancing energy efficiency and renewable energy use in Brazil. Energy Policy, 32(12), 1437-1450. http://doi.org/10.1016/S0301-4215(03)00122-8

George, M., \& Banerjee, R. (2009). Analysis of impacts of wind integration in the Tamil Nadu grid. Energy Policy, 37(9), 3693-3700. http://doi.org/10.1016/j.enpol.2009.04.045

GNESD. (2014). Renewable energy-based rural electrification: The Mini-Grid Experience from India. New Delhi: Prepared by The Energy and Resources Institute (TERI) for the Global Network on Energy for Sustainable Development (GNESD).

Government of India. Minister of Finance Union Budget 2017-18 Budget Speech. Retrieved from http://indiabudget.nic.in/ub2017-18/bs/bs.pdf

Hiremath, R. B., Kumar, B., Balachandra, P., Ravindranath, N. H., \& Raghunandan, B. N. (2009). Decentralised renewable energy: Scope, relevance and applications in the Indian context. Energy for Sustainable Development, 13(1), 4-10. http://doi.org/10.1016/j.esd.2008.12.001

IFC. (2012). Lighting Asia: Solar Off-Grid Lighting, Market Analysis of India, Bangladesh, Nepal, Pakistan, Indonesia, Cambodia and Philippines. from http://www.ifc.org/wps/wcm/connect/311790804b5ca8d783fbc3bbd578891b/Lighting-Asia-offgrid-lighting -Report072512.pdf?MOD=AJPERES

Johnson, V. C., \& Hall, S. (2014). Community energy and equity: The distributional implications of a transition to a decentralized electricity system People, Place and Policy (2014): 8/3, pp. 149-167. http://doi.org/10.3351/ppp.0008.0003.0002

Kanase-Patil, A. B., Saini, R. P., \& Sharma, M. P. (2011). Sizing of integrated renewable energy system based on load profiles and reliability index for the state of Uttarakhand in India. Renewable Energy, 36(11), 2809-2821. http://doi.org/10.1016/j.renene.2011.04.022

Kapoor, K. K., \& Dwivedi, Y. K. (2017). A Take on Solar Power in India. Economic \& Political Weekly, 52(7), 
21.

Kapoor, K., Pandey, K. K., Jain, A. K., \& Nandan, A. (2014). Evolution of solar energy in India: A review. Renewable and Sustainable Energy Reviews, 40, 475-487. http://doi.org/10.1016/j.rser.2014.07.118

Kaundinya, D. P., Balachandra, P., \& Ravindranath, N. H. (2009). Grid-connected versus stand-alone energy systems for decentralized power-A review of literature. Renewable and Sustainable Energy Reviews, 13(8), 2041-2050. http://doi.org/10.1016/j.rser.2009.02.002

Khare, V., Nema, S., \& Baredar, P. (2013). Status of solar wind renewable energy in India. Renewable and Sustainable Energy Reviews, 27, 1-10. http://doi.org/10.1016/j.rser.2013.06.018

Kumar, A., Kumar, K., Kaushik, N., Sharma, S., \& Mishra, S. (2010). Renewable energy in India: current status and future potentials. Renewable and Sustainable Energy Reviews, 14(8), 2434-2442. http://doi.org/10.1016/j.rser.2010.04.003

Kumar, A., Mohanty, P., Palit, D., \& Chaurey, A. (2009). Approach for standardization of off-grid electrification projects. Renewable and Sustainable Energy Reviews, 13(8), 1946-1956. http://doi.org/10.1016/j.rser.2009.03.008

Lok Sabha. (2015). Sixth Report Standing Committee on Energy (2014-15) (Sixteenth Lok Sabha) Ministry of New and Renewable Energy Demands For Grants (2015-16) Presented to Lok Sabha on 27.04.2015

Madrigal \& Porter. (2013). Operating and Planning Electricity Grids with Variable Renewable Generation: Review of Emerging Lessons from Selected Operational Experiences and Desktop Studies. Washington, DC: World Bank. http://doi.org/10.1596/978-0-8213-9734-3.

Mani, S., \& Dhingra, T. (2013). Offshore wind energy policy for India-Key factors to be considered. Energy Policy, 56, 672-683 http://doi.org/10.1016/j.enpol.2013.01.031

Ministry of Environment \& Forests. (MoEF, 2015). India's first Biennial Report to UNFCC. Retrieved from http://www.moef.gov.in/sites/default/files/indbur1.pdf

Ministry of New and Renewable Energy. (2015a). Achievements and Initiatives of Ministry of Power, Coal, and Renewable Energy. Retrieved November, 2015, from http://mnre.gov.in/file-manager/UserFiles/Achievements-of-Power-Coal-and-MNRE-Nov2015-English.pdf

Ministry of New and Renewable Energy. (2015b). Annual Report 2014-15. Retrieved from http://mnre.gov.in/file-manager/annual-report/2014-2015/EN/index.htm

Ministry of Power. (2017). National Electricity Policy. Retrieved from http://powermin.nic.in/en/content/national-electricity-policy

Mishra, A., \& Sarangi, G. K. (2011). Off-grid energy development in India: an approach towards sustainability. OASYS-South Asia Project Working Paper.

Nouni, M. R., Mullick, S. C., \& Kandpal, T. C. (2008). Providing electricity access to remote areas in India: An approach towards identifying potential areas for decentralized electricity supply. Renewable and Sustainable Energy Reviews, 12, 1187-1220 http://doi.org/10.1016/j.rser.2007.01.008

Painuly, J. P. (2001). Barriers to renewable energy penetration; a framework for analysis. Renewable energy, 24(1), 73-89. http://doi.org/10.1016/S0960-1481(00)00186-5

Palit, D. (2013). Solar energy programs for rural electrification: Experiences and lessons from South Asia. Energy for Sustainable Development, 17(3), 270-279. http://doi.org/10.1016/j.esd.2013.01.002

Palit, D., \& Chaurey, A. (2011). Off-grid rural electrification experiences from South Asia: Status and best practices. Energy for Sustainable Development, 15(3), 266-276. http://doi.org/10.1016/j.esd.2011.07.004

Palit, D., Garimella, A., Shardul, M., \& Chaudbury, S. (2015). TERI. Analysis of the electrification programme in India using the 'energy plus' framework and the key lessons. New Delhi: The Energy and Resources Institute. $81 \mathrm{pp}$.

Pandey, S., Singh, V. S., Gangwar, N. P., Vijayvergia, M. M., Prakash, C., \& Pandey, D. N. (2012). Determinants of success for promoting solar energy in Rajasthan, India. Renewable and Sustainable Energy Reviews, 16(6), 3593-3598. http://doi.org/10.1016/j.rser.2012.03.012

PIB. (2015). Press Release Revision of cumulative targets under National Solar Mission from 20,000 MW by 2021-22 to 1,00,000 MW. Retrieved from http://pib.nic.in/newsite/PrintRelease.aspx?relid=122566 
Raman, P., Murali, J., Sakthivadivel, D., \& Vigneswaran, V. S. (2012). Opportunities and challenges in setting up solar photo voltaic based micro grids for electrification in rural areas of India. Renewable and Sustainable Energy Reviews, 16(5), 3320-3325. http://doi.org/10.1016/j.rser.2012.02.065

Reddy, B. S., \& Srinivas, T. (2009). Energy use in Indian household sector-An actor-oriented approach. Energy, 34(8), 992-1002. http://doi.org/10.1016/j.energy.2009.01.004

Rogers, J. C., Simmons, E. A., Convery, I., \& Weatherall, A. (2012). Social impacts of community renewable energy projects: findings from a woodfuel case study. Energy Policy, 42, 239-247. $\mathrm{http}: / /$ doi.org/10.1016/j.enpol.2011.11.081

Rolland, S., \& Glania, G. (2011). Hybrid mini-grids for rural electrification: lessons learned. Alliance for Rural Electrification (ARE), Brussels, Belgium, Mar. Retrieved from http://www.ruralelec.org/fileadmin/DATA/Documents/06_Publications/Position_papers/ARE_Mini-grids__Full_version.pdf

Ruggiero, S., Onkila, T., \& Kuittinen, V. (2014). Realizing the social acceptance of community renewable energy: A process-outcome analysis of stakeholder influence. Energy Research \& Social Science, 4, 53-63. http://doi.org/10.1016/j.erss.2014.09.001

Samanta, K. P. (2015). A Study of Rural Electrification Infrastructure in India. Journal of Business and Management, 17(2).

Sargsyan, G., Bhatia, M., Banerjee, S. G., \& Raghunathan, K. (2011). Unleashing the potential of renewable energy in India. World Bank, Washington, D.C. https://doi.org/10.1596/978-0-8213-8780-1

Schmid, G. (2012). The development of renewable energy power in India: Which policies have been effective? Energy Policy, 45, 317-326. http://doi.org/10.1016/j.enpol.2012.02.039

Sen, R., \& Bhattacharyya, S. C. (2014). Off-grid electricity generation with renewable energy technologies in India: An application of HOMER. Renewable Energy, 62, 388-398. http://doi.org/10.1016/j.renene.2013.07.028

Smith, K. R., \& Sagar, A. (2014). Making the clean available: Escaping India's chulha trap. Energy Policy, 75, 410-414. http://doi.org/10.1016/j.enpol.2014.09.024

Sood, V. K., Fischer, D., Eklund, J. M., \& Brown, T. (2009, October). Developing a communication infrastructure for the smart grid. In Electrical Power \& Energy Conference (EPEC), 2009 IEEE (pp. 1-7). IEEE. https://doi.org/10.1109/EPEC.2009.5420809

Tenenbaum, B., Chris G., Tilak S., \& James K. (2014). From the Bottom Up: How Small Power Producers and Mini-Grids Can Deliver Electrification and Renewable Energy in Africa. Directions in Development. Washington, DC: World Bank.

Union Budget of India 2015-16. Retrieved from http://pib.nic.in/budget2015/budgetdoc/gbEngRelease.pdf

Union Budget of India 2016-17. Retrieved from http://pib.nic.in/eventsite/budget2016/pdf/budspeecheng.pdf

Van Der Schoor, T., \& Scholtens, B. (2015). Power to the people: Local community initiatives and the transition to sustainable energy. Renewable and Sustainable Energy Reviews, 43, 666-675. http://doi.org/10.1016/j.rser.2014.10.089

Walker, B. J., Wiersma, B., \& Bailey, E. (2014). Community benefits, framing and the social acceptance of offshore wind farms: an experimental study in England. Energy Research \& Social Science, 3, 46-54. http://doi.org/10.1016/j.erss.2014.07.003

Walker, G., Hunter, S., Devine-Wright, P., Evans, B., \& Fay, H. (2007). Harnessing community energies: explaining and evaluating community-based localism in renewable energy policy in the UK. Global Environmental Politics, 7(2), 64-82. http://doi.org/10.1162/glep.2007.7.2.64

Walker, G., Simcock, N., \& Smith, S. J. (2012). Community Energy Systems. International Encyclopedia of Housing and Home, 1, 194-198.

World Bank. (2008). Residential Consumption of Electricity in India: Documentation of Data and Methodology. Background Paper for India: Strategies for Low Carbon Growth. 


\section{Notes}

Note 1. These include decentralized power generation, or off-grid solutions, and standalone systems for households.

Note 2. A village is deemed electrified, if $10 \%$ of all the households of the village has electricity access and if electricity provided to public spaces such as schools, panchayat officers, health centres, community centres and dispensaries. (Samanta, 2015).

Note 3.90 percent of the project equipment and other costs are provided by the Ministry, and a proportion of the remaining cost by the state government.

\section{Copyrights}

Copyright for this article is retained by the author(s), with first publication rights granted to the journal.

This is an open-access article distributed under the terms and conditions of the Creative Commons Attribution license (http://creativecommons.org/licenses/by/4.0/). 